\title{
Interaction of Insular Cortex and Ventral Striatum Mediates the Effect of Incentive Memory on Choice Between Goal-Directed Actions
}

\author{
Shauna L. Parkes, Laura A. Bradfield, and Bernard W. Balleine \\ Brain and Mind Research Institute, The University of Sydney, Sydney, New South Wales 2050, Australia
}

The anterior insular cortex (IC) and the nucleus accumbens (NAc) core have been separately implicated in the selection and performance of actions based on the incentive value of the instrumental outcome. Here, we examined the role of connections between the IC and the NAc core in the performance of goal-directed actions. Rats were trained on two actions for distinct outcomes, after which one of the two outcomes was devalued by specific satiety immediately before a choice extinction test. We first confirmed the projection from the IC to the NAc core and then disconnected these structures via asymmetrical excitotoxic lesions before training. Contralateral, but not ipsilateral, disconnection of the IC and NAc core disrupted outcome devaluation. We hypothesized that communication between the IC and NAc core is necessary for the retrieval of incentive value at test. To test this, we infused the $\mathrm{GABA}_{\mathrm{A}}$ agonist muscimol into the IC and the $\mu$-opioid receptor antagonist CTAP into the contralateral NAc before the choice extinction test. As expected, inactivation of the IC in one hemisphere and blocking $\mu$-opioid receptors in the contralateral NAc core abolished outcome-selective devaluation. These results suggest that the IC and NAc core form part of a circuit mediating the retrieval of outcome values and the subsequent choice between goal-directed actions based on those values.

Key words: goal-directed action; gustatory cortex; nucleus accumbens; outcome devaluation; value-based decision-making

\section{Introduction}

The ability to acquire and exert control over reward-related actions is a fundamental process that requires the agent to encode the relationship between an action and its outcome. However, it has long been recognized that knowledge of the action-outcome association alone is not sufficient to determine the performance of an action; in addition, the subject must evaluate the incentive value of the outcome (Dickinson and Balleine, 1994; Balleine and Dickinson, 1998). Importantly, this value is not fixed, but rather changes depending on the prevailing needs and desires of the subject. Appropriate decision-making necessitates, therefore, that the agent also encode changes in the value of the outcome and, moreover, that the current value of the outcome is retrieved to guide behavior.

Recent work in rats has shown that the anterior insular cortex (IC) is critical for the retrieval of instrumental outcome values. Rats with bilateral lesions of the IC are insensitive to changes in the incentive value of the outcome, but only when assessed in

Received Oct. 7, 2014; revised Jan. 20, 2015; accepted Feb. $27,2015$.

Author contributions: S.L.P. and B.W.B. designed research;S.L.P. and L.A.B. performed research;S.L.P. and L.A.B. analyzed data; S.L.P., L.A.B., and B.W.B. wrote the paper.

This work was supported by the National Institute of Mental Health-National Institutes of Health (Grant MH56446 to B.W.B.), the National Health and Medical Research Council (Grant 633267 to B.W.B.), and the Australian Research Council (Laureate Fellowship FL0992409 to B.W.B.).

The authors declare no competing financial interests.

Correspondence should be addressed to Bernard W. Balleine, Brain and Mind Research Institute, University of Sydney, Level 6, 94 Mallett Street, Camperdown, NSW 2050, Australia. E-mail: bernard.balleine@sydney.edu.au.

DOI:10.1523/JNEUROSCI.4153-14.2015

Copyright $\odot 2015$ the authors $\quad 0270-6474 / 15 / 356464-08 \$ 15.00 / 0$ extinction (Balleine and Dickinson, 2000). Similarly, outcome devaluation is disrupted by infusion of the NMDA receptor antagonist ifenprodil into the IC immediately before a choice extinction test (Parkes and Balleine, 2013). Based on these data, we have recently proposed that encoding changes in outcome value is determined by a circuit involving connections between the BLA and the IC, whereas subsequent retrieval of these values appears to depend on the IC, which serves to guide choice between instrumental actions (Parkes and Balleine, 2013). However, it remains open to question how the information coded in the IC makes contact with the broader instrumental conditioning circuit to drive performance. A likely candidate is connections between the IC and the ventral striatum (Allen et al., 1991; Chikama et al., 1997; Naqvi and Bechara, 2009, 2010), particularly with the core region of the nucleus accumbens (NAc) (Wright and Groenewegen, 1996; Reynolds and Zahm, 2005). Considerable evidence has implicated the core in the selection and performance of actions based on the incentive value of the outcome (Corbit et al., 2001; Shiflett and Balleine, 2010; Burton et al., 2015), which a recent study suggests is dependent on $\mu$-opioid receptor-related process in the core (Laurent et al., 2012). It seems reasonable to propose, therefore, that IC-core connections may mediate the retrieval and performance of actions based on incentive memory. To test this hypothesis, we first confirmed the connection between the IC and the NAc core using the retrograde tracer Fluorogold (FG; Fluorochrome) and then disconnected these structures before training to determine whether communication between them is necessary for the performance of goal-directed actions. Next, we 
used a pharmacological disconnection procedure to determine precisely the temporal involvement of the IC-core pathway in changes in choice between goal-directed actions after a change in outcome value.

\section{Materials and Methods}

Experiment 1: Effect of pretraining, excitotoxic-lesion-induced disconnection of the IC from the NAc core on outcome devaluation

First, we confirmed the connection between the IC and the NAc core (Allen et al., 1991; Wright and Groenewegen, 1996; Chikama et al., 1997; Reynolds and Zahm, 2005; Naqvi and Bechara, 2010, 2009) using retrograde tracing. Rats $(n=3)$ were injected unilaterally with the retrograde neuronal tracer FG and both the ipsilateral and contralateral IC were examined for labeled cells. Next, we examined whether the connection between the IC and the NAc core is necessary for choice between goaldirected actions. Rats received either sham or excitotoxic, ipsilateral, or contralateral lesions of the IC and NAc core before behavioral training.

Subjects and apparatus. Forty experimentally naive male outbred hooded Wistar rats $(300-350 \mathrm{~g})$ served as subjects in the lesion experiment. They were housed in plastic boxes ( $2-3$ rats per box) located in a climate-controlled colony room and were maintained on a $12 \mathrm{~h}$ light/ dark cycle. Rats were allowed $3-5 \mathrm{~d}$ to recover from surgery, during which time they were handled and weighed daily. Five days before the behavioral procedures, the rats were put on a food deprivation schedule to maintain them at $\sim 85 \%$ of their ad libitum feeding weight. All experimental procedures were approved by the Animal Ethics Committee at the University of Sydney.

Training and testing took place in 16 MED Associates operant chambers enclosed in sound- and light-resistant shells. Each chamber was equipped with a pump that was fitted with a syringe that delivered $20 \%$ sucrose solution $(0.1 \mathrm{ml})$ and a pellet dispenser that delivered grain pellets (45 mg; Bioserv Biotechologies) into a recessed magazine when activated. The chambers contained two retractable levers that could be inserted to the left and the right of the magazine. An infrared photobeam crossed the magazine opening, allowing for the detection of head entries. A house light $(3 \mathrm{~W}, 24 \mathrm{~V})$ provided illumination of the operant chamber. A set of two microcomputers running MED Associates proprietary software (Med-PC) controlled all experimental events and recorded lever presses and magazine entries.

Surgery. Rats were anesthetized using isoflurane (Laser Animal Health) mixed with oxygen and were mounted on a stereotaxic apparatus (David Kopf Instruments). Rats were subcutaneously injected with 0.1 $\mathrm{ml}$ solution of rimadyl and $0.1 \mathrm{ml}$ solution of bupivacaine. An incision was then made to expose the scalp and the incisor bar was adjusted to a flat skull position. Excitotoxic lesions were made by infusing $0.8 \mu \mathrm{l}$ for IC lesions (anteroposterior $+1.0 \mathrm{~mm}$; mediolateral $\pm 4.0 \mathrm{~mm}$ at an angle of $6^{\circ}$; dorsoventral: $-7.5 \mathrm{~mm}$ from skull surface) or $0.4 \mu \mathrm{l}$ for NAc core lesions (anteroposterior $+1.4 \mathrm{~mm}$; mediolateral \pm 2.1 ; dorsoventral: $-7.2 \mathrm{~mm}$ from skull surface) of NMDA $(10 \mathrm{mg} / \mathrm{ml})$ in sterilized $0.1 \mathrm{M}$ PBS, pH 7.2 over 8 minutes (IC lesion) or 4 minutes (core lesion). The needle was left in place for an additional 5 minutes before removal to allow for diffusion. All coordinates given are distance from bregma according to the rat brain atlas of Paxinos and Watson (2007). Lesions were either made in the same hemisphere (group Ipsi) or in contralateral hemispheres (group Contra). The hemisphere of each infusion was counterbalanced across rats. That is, for half of the rats in group Ipsi, the lesions were located in the right hemisphere, whereas for the remaining rats, the lesions were located in the left hemisphere. Similarly, in group Contra, for half of the rats, the lesion in the IC was located in the right hemisphere and the lesion in the NAc core was located in the left hemisphere and vice versa for the remaining rats. Sham-operated rats underwent the same procedure except that only sterilized PBS was infused. Immediately after the surgical procedure, all rats were injected intraperitoneally with a prophylactic $(0.3 \mathrm{ml})$ dose of $300 \mathrm{mg} / \mathrm{kg}$ solution of procaine penicillin. Rats were allowed $5 \mathrm{~d}$ to recover from surgery, during which time they were handled and weighed daily before food restriction and training.

To confirm and visualize the neural connections between the IC and the NAc core, we also injected a retrograde neuronal tracer into the NAc core in a separate group of rats $(n=3)$. Rats were placed in the stereotaxic frame and prepared as above with $100 \mathrm{nl}$ of 3\% FG (Fluorochrome) solution diluted in $0.9 \%$ saline injected unilaterally in the NAc core (anteroposterior $+1.4 \mathrm{~mm}$; mediolateral \pm 2.1 ; dorsoventral: $-7.2 \mathrm{~mm}$ from skull surface). The injector was left in place for 5 minutes to allow diffusion before being retracted. Immediately after the surgical procedure, rats were injected intraperitoneally with a prophylactic $(0.3 \mathrm{ml})$ dose of $300 \mathrm{mg} / \mathrm{kg}$ solution of procaine penicillin.

Histology. Rats injected with the retrograde tracer were rapidly and deeply anesthetized with sodium pentobarbital and perfused transcardially with ice-cold $4 \%$ paraformaldehyde in $0.1 \mathrm{~m}$ phosphate buffer 1 week after surgery. Brains were removed and postfixed in $4 \%$ paraformaldehyde overnight. Subsequently, $30 \mu \mathrm{m}$ coronal sections were cut using a vibratome (Leica). Every fourth section was collected to form a series and then mounted onto slides and coverslipped with Fluoromount-G (Southern Biotech).

Rats that received lesions were deeply anesthetized with a $0.3 \mathrm{ml}$ intraperitoneal injection of sodium pentobarbital and perfused transcardially with ice-cold $4 \%$ paraformaldehyde in $0.1 \mathrm{~m}$ phosphate buffer after behavioral training. Brains were stored in $4 \%$ formaldehyde for $1 \mathrm{~h}$ and were then transferred to a $20 \%$ sucrose solution in phosphate buffer saline, $\mathrm{pH}$ 7.2. The brains were sectioned coronally at $40 \mu \mathrm{m}$ through the IC and the NAc core. Every third section was collected on a slide and stained with cresyl violet. The location and size of the lesion was determined under a microscope by a trained observer unaware of the subjects' group designations using the boundaries defined by the atlas of Paxinos and Watson (2007). Subjects with inaccurate lesions were excluded from the statistical analysis.

\section{Behavioral procedures}

Instrumental training. After $5 \mathrm{~d}$ of food deprivation, rats were given 2 sessions of magazine training to familiarize them with entering the magazine for the outcomes. Rats were confined to the operant chamber while the two outcomes (grain pellets and sucrose solution) were delivered at random $60 \mathrm{~s}$ intervals. Forty outcomes were delivered per session, 20 of each outcome. Rats then received $9 \mathrm{~d}$ of instrumental training, during which time 2 responses (left and right lever presses) were trained with the 2 different outcomes in the same session. During the session, each lever was presented twice for a maximum of 10 minutes each or until 20 outcomes were earned; that is, rats could earn a maximum of 40 grain pellets and 40 sucrose deliveries within the session. The intertrial interval between lever presentations was 2.5 minutes. The order of the lever presentation was alternated and counterbalanced across rats and days. Response-outcome relationships were also counterbalanced across rats. For the first $3 \mathrm{~d}$, lever pressing was continuously reinforced; then, the probability of the outcome given a response was gradually shifted over days using increasing random ratio (RR) schedules. An RR5 schedule ( probability of receiving an outcome given a response $=0.2$ ) was used on days 4-6 and an RR10 (probability of receiving an outcome given a response $=0.1$ ) schedule was used on days 7-9.

Outcome devaluation. Twenty-four hours after the final instrumental training session, rats were given an outcome devaluation test. In this test, rats received ad libitum access to 1 of the 2 outcomes for $1 \mathrm{~h}$ in clear plastic feeding cages located in a different room than that used for training. Half of the rats in each response-outcome assignment received grain pellets and the remaining rats received sucrose solution. Immediately after devaluation, rats were given a 3 minute choice extinction test in which both levers were available but no outcome was delivered. Testing in extinction ensured that the rats had to recall both the current action-outcome contingencies and the current values of the two instrumental outcomes. All rats were given $1 \mathrm{~d}$ of rest and, $48 \mathrm{~h}$ after the first outcome devaluation test, all rats were given a second test with the other outcome; that is, rats that were pre-fed on grain pellets in the first devaluation test were now pre-fed with sucrose solution and vice versa.

Consumption test of specific satiety. To assess the ability of lesioned rats to distinguish between the two outcomes and to determine whether the devaluation procedure was effective, rats were given a consumption version of the specific satiety devaluation test. One of the two instrumental 
outcomes was devalued in the same manner as in the outcome devaluation tests described above and rats were then given access to both outcomes for 10 minutes. The total amount consumed of each outcome (valued and devalued) was recorded. The amount of sucrose consumed in milliliters was converted to grams by multiplying by 1.05 ( $1 \mathrm{~L}$ of sucrose solution weighs $1050 \mathrm{ml}$ ). Forty-eight hours later, rats were given a second consumption test with the other outcome; again, rats that were pre-fed on grain pellets in the first devaluation test were now pre-fed with sucrose solution and vice versa.

Data analyses. All analyses were conducted using a mixed-model ANOVA followed by simple main effects analyses to establish the source of any significant interactions. Statistical significance was set at $p \leq 0.05$. Data are presented as mean \pm SEM.

\section{Experiment 2: Effect of pharmacological disconnection of IC and $N A c$ core induced immediately before test}

Experiment 1 confirmed that a functional connection between the IC and NAc core is required for goal-directed behavior. The aim of this experiment was to determine the temporal role of these connections. Rats were implanted with a unilateral cannula targeting the IC and a unilateral cannula targeting the NAc core. Cannulae were either implanted in the same hemisphere or in contralateral hemispheres.

Subjects and apparatus. Forty-three experimentally naive male outbred hooded Wistar rats $(300-350 \mathrm{~g})$ served as subjects. The housing conditions and training apparatus were identical to those described above.

Surgery. Rats were anesthetized and placed in the stereotaxic frame as described above. Then, rats were implanted with a 26 gauge unilateral guide cannula (Plastics One) directed toward the IC and a 26 gauge unilateral guide cannula directed toward the NAc core before behavioral testing. Cannulae were either implanted in the same hemisphere (group Ipsi) or in contralateral hemispheres (group Contra). The hemisphere of each cannula was counterbalanced across rats. The coordinates used for the IC were as follows: anteroposterior $+1.0 \mathrm{~mm}$; mediolateral $\pm 4.0 \mathrm{~mm}$ (angle $6^{\circ}$ ); dorsoventral $-6.5 \mathrm{~mm}$ (from skull surface). The coordinates used for the core in group Contra were as follows: anteroposterior +1.4 $\mathrm{mm}$; mediolateral \pm 2.1 ; dorsoventral: $-6.2 \mathrm{~mm}$ (from skull surface). The coordinates used for the core in group Ipsi were as follows: anteroposterior $+3.4 \mathrm{~mm}\left(\right.$ angle $\left.16^{\circ}\right)$; mediolateral \pm 2.1 ; dorsoventral: -7.0 $\mathrm{mm}$ (from skull surface). All coordinates given are the distance from bregma according to the rat brain atlas of Paxinos and Watson (2007). Different coordinates were required for the cannulae targeting the NAc core in the ipsilateral versus contralateral animals due to the close proximity of the NAc core and the IC cannulae in the ipsilateral animals. The guide cannulae were maintained in position with dental cement and dummy cannulae were kept in each guide at all times except during microinfusions. Immediately after the surgical procedure, all rats were injected intraperitoneally with a prophylactic $(0.3 \mathrm{ml})$ dose of $300 \mathrm{mg} / \mathrm{kg}$ solution of procaine penicillin. Rats were allowed $5 \mathrm{~d}$ to recover from surgery, during which time they were handled and weighed daily before food restriction and training.

Drugs and microinfusions. CTAP (Sigma-Aldrich), a selective $\mu$-opioid receptor antagonist, and muscimol (Sigma-Aldrich), a $\mathrm{GABA}_{\mathrm{A}}$ receptor agonist, were dissolved in $0.9 \%(\mathrm{w} / \mathrm{v})$ nonpyrogenic saline to obtain a final concentration of $2 \mu \mathrm{g} / \mu \mathrm{l}$ (Soderman and Unterwald, 2008; Trezza et al., 2011; Laurent et al., 2012) and $0.5 \mu \mathrm{g} / \mu \mathrm{l}$ (LaGraize and Fuchs, 2007), respectively. Nonpyrogenic saline infusions were used as the vehicle solution. Rats were either given a unilateral infusion of muscimol (into the IC) and a unilateral infusion of CTAP (into the core) or infusions of vehicle (into the IC and the core). Solutions were infused by inserting a 33 gauge internal cannula into the guide cannula. The internal cannula was connected a $25 \mu$ l glass syringe attached to infusion pumps (Harvard Apparatus) and projected an additional $1 \mathrm{~mm}$ ventral to the tip of the guide cannula. A total volume of $0.3 \mu \mathrm{l}$ was delivered at a rate of 0.1 $\mu \mathrm{l} / \mathrm{min}$ for infusions into the NAc core and $0.5 \mu \mathrm{l}$ was delivered at a rate of $0.25 \mu \mathrm{l} / \mathrm{min}$ for infusions into the IC. The internal cannula remained in place for a further 1 minute after the infusions and was then removed. One day before infusions, the dummy cannulae were removed and the infusion pumps were turned on for 3-5 minutes to familiarize the rats with the procedure.
Histology. Subsequent to behavioral testing, subjects received a lethal dose of sodium pentobarbital. The brains were removed and sectioned coronally at $40 \mu \mathrm{m}$ through the IC and the NAc core. Every third section was collected on a slide and stained with cresyl violet. The location of cannula tips was determined under a microscope by a trained observer unaware of the subjects' group designations using the boundaries defined by the atlas of Paxinos and Watson (2007). Subjects with inaccurate cannula placement were excluded from the statistical analysis.

\section{Behavioral procedures}

Methods for magazine training and instrumental training were identical to those described for Experiment 1.

Outcome devaluation test. Rats were given $1 \mathrm{~h}$ ad libitum access to 1 of the 2 outcomes in clear plastic feeding cages and were then given either a unilateral infusion of muscimol (into the IC) and a unilateral infusion of CTAP (into the core) or a vehicle infusion (into the IC and the core). Twenty minutes later, rats were placed in the operant chambers and given a 5 minute choice extinction test. All rats were then given $1 \mathrm{~d}$ of rest and then a second outcome devaluation test with the same outcome. Rats that had previously received a vehicle infusion now received muscimol and CTAP, whereas rats that had previously received muscimol and CTAP now received vehicle infusions.

Consumption test of specific satiety. To assess the ability of rats to distinguish between the two outcomes after infusion of CTAP and muscimol, rats were given a consumption test of specific satiety-induced outcome devaluation. The test was identical to that described for Experiment 1 except that rats were infused with either muscimol and CTAP or vehicle immediately after the devaluation and before the choice consumption test. Forty-eight hours later, rats were given a second consumption test. Rats that had previously received a vehicle infusion now received muscimol and CTAP, whereas rats that had previously received muscimol and CTAP now received a vehicle infusion.

Data analyses. All analyses were conducted using a mixed-model ANOVA followed by simple main effects analyses to establish the source of any significant interactions. Statistical significance was set at $p \leq 0.05$. Data are presented as mean \pm SEM. Rats in Experiment 2 had grain or sucrose devalued in each test, not both outcomes as in Experiment 1, so instrumental test data are presented as a percentage of baseline responding to control for any baseline differences in responding for grain versus sucrose reward.

\section{Results}

\section{Experiment 1: Pretraining excitotoxic lesion disconnection of the IC and NAc core disrupts specific satiety-induced outcome devaluation \\ Histology}

Figure 1 shows a representative example of the FG injection site in the left NAc core (Fig. 1A) and the pattern of FG that was retrogradely transported to the contralateral (Fig. 1C) and ipsilateral (Fig. 1D) IC. The rostral-caudal spread of the FG injection for all animals is shown in Figure $1 B$. Consistent with previous literature, considerably more retrograde labeling was observed in the ipsilateral IC than in the contralateral IC (Wright and Groenewegen, 1996; Reynolds and Zahm, 2005; Allen et al., Naqvi and Bechara, 2009, 2010). Histological verification of the lesion placements are presented in Figure 2, which shows the location and size of the lesion for ipsilateral (left) and contralateral (right) placements. The lesions targeted the areas of the IC and NAc core that were identified from the results of our retrograde tracing. Ten rats were excluded because of incorrect location or size of the lesion, yielding the following group sizes: group Sham, $n=11$; group Ipsi, $n=9$; and group Contra, $n=10$.

\section{Behavioral results}

Lever-pressing performance increased across instrumental training and did not differ between groups (Fig. $3 A$ ). Statistical analyses confirmed a significant effect of session $\left(F_{(1,27)}=267.8, p<\right.$ $0.05)$, but no effect of group nor any interaction between these 

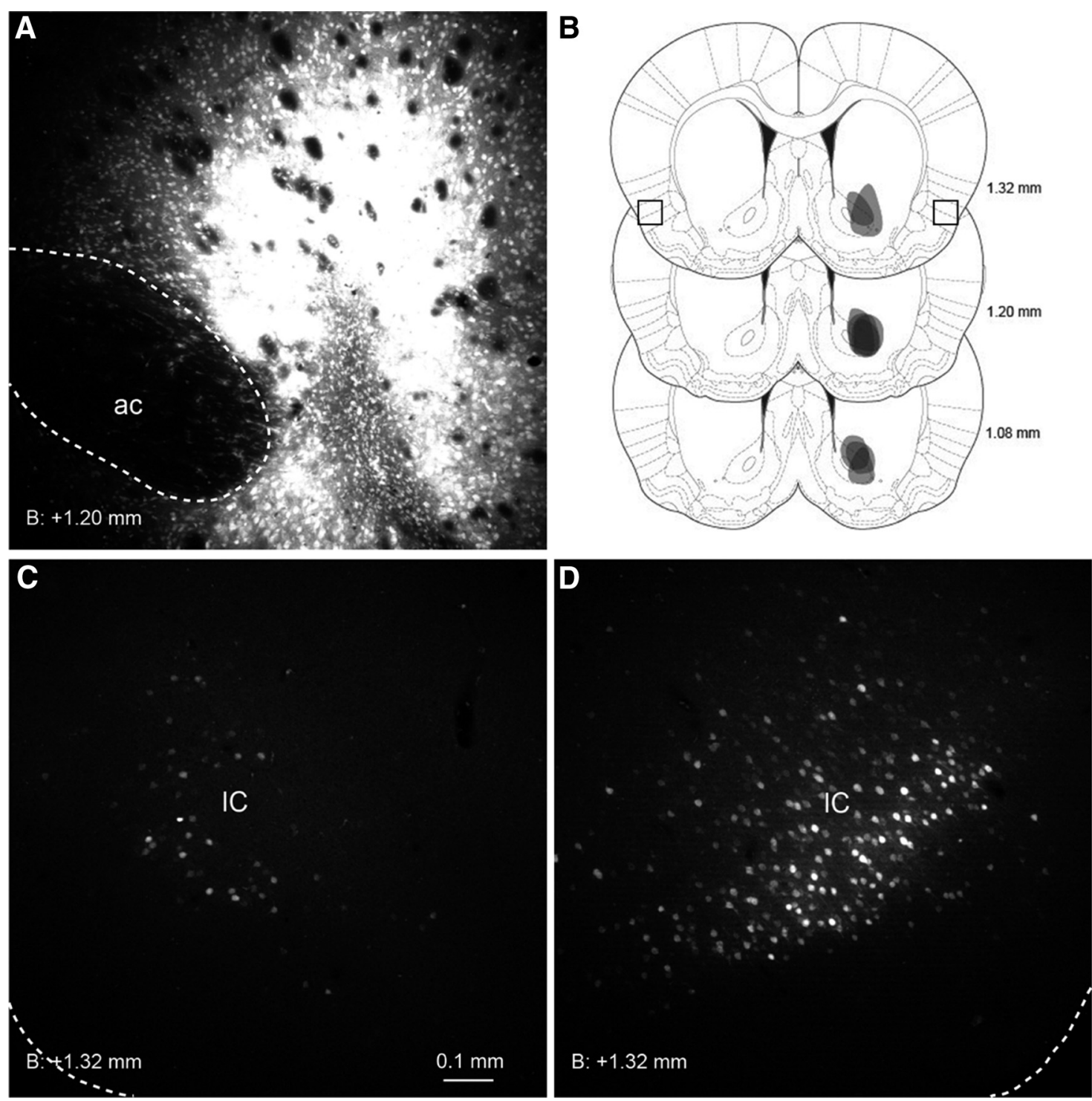

Figure 1. FG injection in the NAc core and retrograde labeling in the IC. $A$, Photomicrograph of a representative FG injection site in the NAc core. ac, Anterior commissure. $B$, Schematic representation (Paxinos and Watson, 2007) of the rostral-caudal spread of the FG injection for all animals $(n=3)$ and the region of the IC imaged for retrograde labeling. $\boldsymbol{C}, \boldsymbol{D}$, Retrograde labeling in the contralateral $(\boldsymbol{C})$ and ipsilateral IC (D).

factors (largest $\left.F_{(1,27)}=1.3, p>0.05\right)$. Performance during the outcome devaluation choice extinction test is presented in Figure $3 B$. Inspection of the figure suggests that sham and ipsilateral lesioned rats pressed the lever associated with the valued outcome more than the lever associated with the devalued outcome. In contrast, rats with contralateral lesions of the IC and NAc core pressed similarly on the valued and devalued levers. Statistical analyses confirmed these observations. A mixed-model ANOVA conducted using factors of lever (valued vs devalued) and group found no effect of group $\left(F_{(1,27)}\right.$ values $\left.<0.1, p>0.05\right)$, indicating the overall rate of lever pressing was similar for ipsilateral, contralateral, and sham lesioned rats. However, there was a significant effect of lever $\left(F_{(1,27)}=35.8, p<0.05\right)$ and a significant group by lever interaction $\left(F_{(1,27)}=4.5, p<0.05\right)$. Simple-effects analyses conducted on the interaction found that rats with sham or ipsilateral lesions pressed significantly more on the valued than the devalued lever $\left(F_{(1,27)}=18.6, p<0.05\right.$, and $F_{(1,27)}=$ $18.7, p<0.05$, respectively), but no such effect was found in rats with contralateral lesions $\left(F_{(1,27)}=3.0, p>0.05\right)$.
This deficit could reflect reduced consumption during the specific satiety treatment; however, the quantity of the pellet or sucrose outcome consumed (in grams) during the satiety devaluation phase did not differ between groups (largest $F_{(1,27)}=0.2, p>0.05$ ). Furthermore, the results of the consumption test do not support such a conclusion nor do they support other alternatives such a reduced outcome discriminability. Those results are shown in Figure $3 C$. Inspection of this figure suggests that rats in all groups consumed more of the valued than the devalued food. That is, after pre-feeding of one of the food outcomes, the rats ate more of a different food than the one just eaten. Importantly, the magnitude of the effect was similar for sham, ipsilateral, and contralateral lesioned rats. Statistical analyses confirmed these observations. A mixed-model ANOVA using factors of food (same vs different) and group revealed a significant effect of food $\left(F_{(1,27)}=112.6, p<0.05\right)$, but no significant effect of group (largest $\left.F_{(1,27)}=1.5, p>0.05\right)$ nor any interaction between these factors (largest $\left.F_{(1,27)}=2.3, p>0.05\right)$. Overall, the quantity (in grams) of the outcome consumed during the devaluation period did not differ between groups (largest $\left.F_{(1,27)}=0.2, p>0.05\right)$. 
Experiment 2: Effect of pharmacological disconnection of IC and NAc core induced immediately before test

Histology

Histological verification of the cannulae placements are presented in Figure 4, which shows the location of the guide cannula tip for ipsilateral (left) and contralateral (right) placements. Fourteen rats were excluded because of incorrect location of one or both of the cannulae, yielding the following group sizes: group Ipsi, $n=15$, and group Contra, $n=14$.

\section{Behavioral results}

Lever-pressing performance increased across instrumental training and did not differ between groups. Statistical analyses confirmed a significant effect of session $\left(F_{(1,27)}=179.0, p<0.05\right)$, but no effect of group nor any interaction between these factors (largest $F_{(1,27)}=2.6, p>0.05$ ). Performance during the outcome devaluation choice extinction test is presented in Figure $5 A$, which shows that rats that received ipsilateral infusions pressed the lever associated with the valued outcome more than the lever associated with the devalued outcome regardless of whether they received a vehicle (group Ipsi-Veh) or drug (group Ipsi-Drug) infusion. Similarly, rats that received vehicle infusions into contralateral hemispheres also showed outcome devaluation (group Contra-Veh). In contrast, rats the received an infusion of muscimol into the IC and CTAP into the contralateral NAc core showed a reduced outcome devaluation effect (group Contra-Drug). Statistical analyses confirmed these observations. A mixed-model ANOVA conducted using factors of group (ipsilateral vs contralateral), lever (valued vs devalued), and infusion type (vehicle vs drug) found no effect of group $\left(F_{(1,27)}=0.2, p>0.05\right)$ or infusion type $\left(F_{(1,27)}=3.1, p>\right.$ $0.05)$, but a significant effect of lever $\left(F_{(1,27)}=7.9, p<0.05\right)$ and a significant interaction between group and lever $\left(F_{(1,27)}=4.6\right.$, $p<0.05)$. There was no main effect of outcome $\left(F_{(1,27)}=2.4, p>\right.$ $0.05)$ and no significant interaction between outcome identity (grain vs sucrose) and lever $\left(F_{(1,27)}=0.2, p>0.05\right)$. Post hoc, 2 -way ANOVAs using factors of lever and group revealed a significant interaction between groups Contra-Drug and Ipsi-Drug $\left(F_{(1,27)}=4.2, p=0.05\right)$. Moreover, a significant interaction was also found between groups Contra-Drug and Contra-Veh $\left(F_{(1,27)}=4.9\right.$, $p<0.05)$, indicating that the devaluation effect also differed in these conditions.

Simple-effects analyses conducted on these interactions found that rats in groups Ipsi-Veh and Ipsi-Drug pressed significantly more on the valued than the devalued lever $\left(F_{(1,27)}=17.1, p<\right.$ 0.05 , and $F_{(1,27)}=4.3, p<0.05$, respectively). Similarly, rats in group Contra-Veh pressed the lever associated with the valued outcome more than the lever associated with the devalued outcome $\left(F_{(1,27)}=5.3, p<0.05\right)$; however, no such effect was found for rats in group Contra-Drug $\left(F_{(1,27)}=0.7, p>0.05\right)$. Finally, the amount of the outcome consumed during the satiety-induced devaluation phase did not differ between the ipsilateral and contralateral groups $\left(F_{(1,27)}=1.0, p>0.05\right)$ or between vehicle- and drug-treated rats $\left(F_{(1,27)}=0.06, p>0.05\right)$ and there was no significant interaction between group and infusion type $\left(F_{(1,27)}=0.2, p>\right.$ 0.05).

The results of the consumption test are shown in Figure $5 B$, which shows that rats in all groups consumed more of the valued than the devalued food. That is, after pre-feeding of one of the food outcomes, the rats ate more of a different food than the one just eaten. Importantly, the magnitude of the effect was similar for all conditions. Statistical analyses confirmed these observations. A mixed-model ANOVA conducted using factors of group (ipsilateral vs contralateral), food (valued vs devalued), and infusion type (vehicle vs drug) revealed a significant effect of food $\left(F_{(1,27)}=68.1, p<0.05\right)$, but no significant effect of drug $\left(F_{(1,27)}\right.$ $<0.01, p>0.05)$ or group $\left(F_{(1,27)}=0.01, p>0.05\right)$ nor any interaction between these factors (largest $F_{(1,27)}=0.7, p>0.05$ ). Moreover, there was no significant interaction between groups Contra-Drug and Ipsi-Drug $\left(F_{(1,27)}<0.1, p>0.05\right)$ nor between 

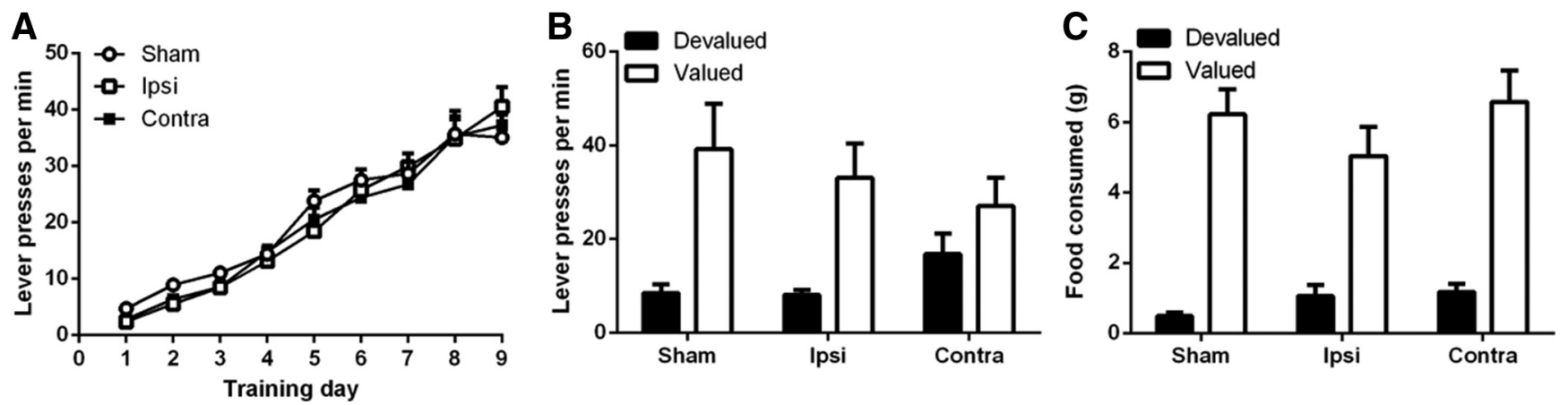

Figure 3. Experiment 1.A, Mean ( + SEM) lever presses per minute across sessions of instrumental training averaged across the two instrumental actions for rats in group Sham (open circles, $n=$ 11), group Ipsi (open squares, $n=9$ ), and group Contra (closed squares, $n=10$ ). $\boldsymbol{B}$, Mean ( + SEM) lever presses per minute during the instrumental choice test. Rats in groups Sham and Ipsi showed increased lever pressing on the lever associated with the valued outcome (white bar) compared with the lever associated with the devalued outcome (black bar). Rats in group Contra pressed equally on both levers. C, Mean (+SEM) food consumed (grams) during the consumption test of specific satiety-induced outcome devaluation. Results are presented separately for consumption of the outcome that was the same as (devalued; black bars) and different from (valued; white bars) the outcome consumed immediately before the test phase.
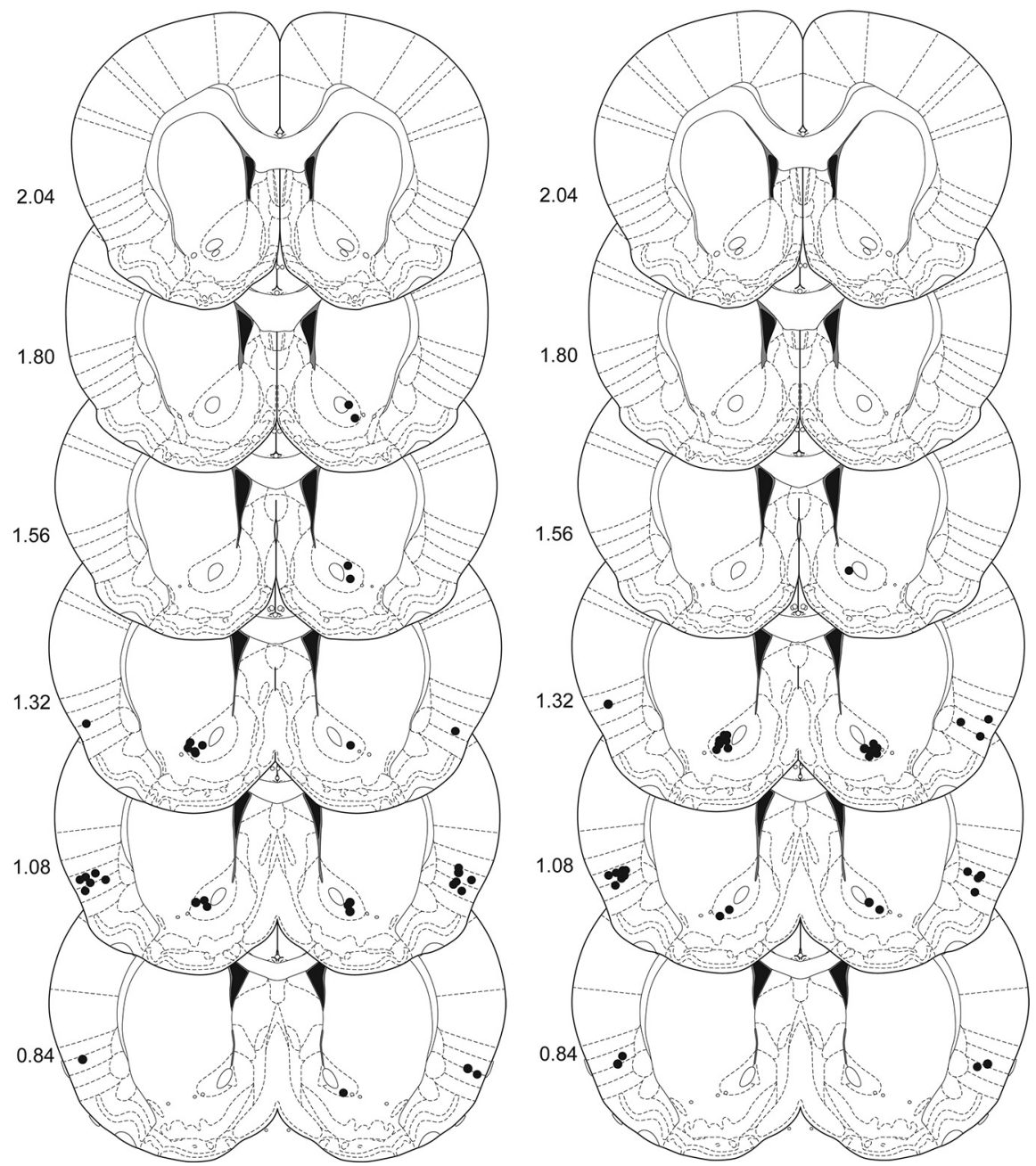

Figure 4. Microinfusion cannula placements as verified on Nissl-stained sections for the IC and the NAc core. Rats were implanted with a unilateral cannula targeting the IC and a unilateral cannula targeting the NAc core. Cannulae were either implanted in ipsilateral (left, $n=12$ ) or contralateral (right, $n=11$ ) hemispheres counterbalanced across hemispheres. Numbers indicate distance (in millimeters) from bregma. Sections are based on Paxinos and Watson (2007).

groups Contra-Drug and Contra-Veh $\left(F_{(1,27)}=0.3, p>0.05\right)$. The quantity of the pellet or sucrose outcome consumed (in grams) during the devaluation period did not differ between the ipsilateral and contralateral groups $\left(F_{(1,27)}=0.05, p>0.05\right)$ or between vehicleand drug-treated rats $\left(F_{(1,27)}=1.0, p>0.05\right)$ and there was no significant interaction between group and infusion type $\left(F_{(1,27)}=0.01, p>0.05\right)$.

\section{Discussion}

The results of the current study provide novel evidence that a functional connection between the IC and the NAc core is required for changes in the value of the instrumental outcome to affect choice between goaldirected actions. First, we demonstrated that disconnection of these structures using excitotoxin-induced lesions before instrumental training attenuated specific satiety-induced outcome devaluation. After outcome devaluation, rats given ipsilateral NMDA-induced or sham lesions pressed the lever associated with the valued outcome more than the lever associated with the devalued outcome. However, rats given contralateral NMDA-induced lesions failed to show this effect. Nevertheless, and most importantly, all rats showed similar effects of specific satiety in consumption; after pre-feeding one of the food outcomes, rats ate more of a different food than the one they had just consumed and to similar degree whether they had received excitotoxic contralateral, ipsilateral, or sham lesions. Therefore, the disconnection did not affect sensitivity to sensory-specific satiety per se nor did it impair the ability to discriminate between the sensory features of the two outcomes, but rather selectively affected instrumental choice performance after a change in outcome value.

To confirm this conclusion, we assessed the effect of disconnecting communication between these structures after devaluation and immediately before the choice test. Rats that received contralateral infusions of the $\mathrm{GABA}_{\mathrm{A}}$ agonist muscimol and the $\mu$-opioid receptor antagonist CTAP into the IC and NAc core, respectively, before the choice test failed to show selective devaluation. In contrast, rats that received ipsilateral infusions of muscimol and 
A

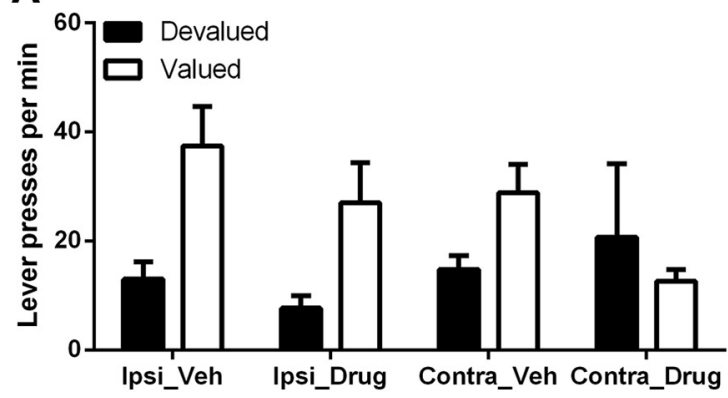

B

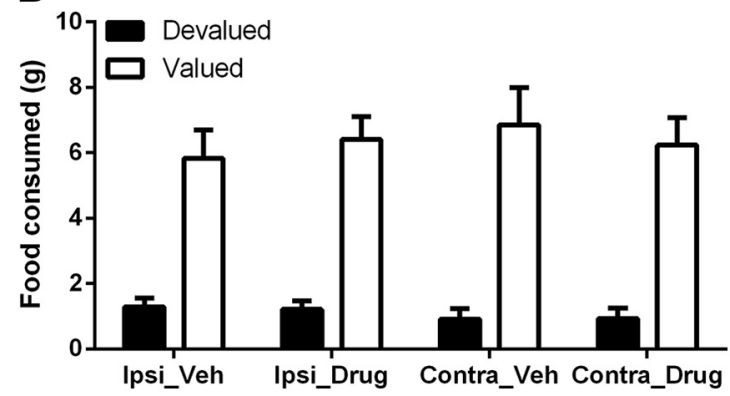

Figure 5. Experiment 2. A, Mean (+SEM) lever presses per minute during the instrumental choice test. Responding is shown as a percentage of baseline responding during the final $2 \mathrm{~d}$ of instrumental training. Rats in Groups Ipsi-Veh, Ipsi-Drug, and Contra-Veh showed increased lever pressing on the lever associated with the valued outcome (white bar) compared with the lever associated with the devalued outcome (black bar). Rats in group Contra-Drug pressed equally on both levers. $\boldsymbol{B}$, Mean ( + SEM) food consumed (grams) during the consumption test of specific satiety-induced outcome devaluation. Results are presented separately for consumption of the outcome that was the same as (devalued; black bars) and different from (valued; white bars) the outcome consumed immediately before the test phase.

CTAP, or rats infused with vehicle, showed selective devaluation. Together, these results provide direct evidence implicating the IC-core pathway in the execution of choice between goaldirected actions based on the current incentive value of the outcome.

Despite the significant impairments observed in rats with compromised IC-core pathways, it appears that our manipulations produced a small, but statistically insignificant, preference for the valued over the devalued lever in Experiment 1. Our tracing results show that the projections from the IC to the NAc are predominately ipsilateral, but contralateral projections do exist. These contralateral projections between the IC and NAc core may have reduced the magnitude of the impairment observed in both experiments. Nevertheless, a number of studies have reported significant deficits in outcome devaluation after disconnections in other regions that share both ipsilateral and weaker contralateral projections; for example, the BLA and the posterior dorsomedial striatum (pDMS) (Kelley et al., 1982; Corbit et al., 2013) and the BLA and the NAc core (McDonald, 1991; Shiflett and Balleine, 2010), suggesting that the ipsilateral pathway is by far the more important for these functional effects. Indeed, this numerical difference was not observed in Experiment 2 using contralateral drug infusions.

The present results are consistent with our previous study showing a role for the IC in the retrieval of incentive memory. Using a sequential disconnection procedure, we found that blocking NMDA receptors in the BLA in one hemisphere before devaluation and in the contralateral IC before test abolished outcome devaluation. In contrast, when NMDA receptor blockade was induced in the reverse order, outcome devaluation remained intact (Parkes and Balleine, 2013). Our results are also consistent with a number of studies that have implicated the core in the performance of goal-directed actions. For example, bilateral lesions of the core impair outcome devaluation and this impairment is not due to an inability to recall the devalued outcome (Corbit et al., 2001, Experiment 1), to discriminate between outcomes (Corbit et al., 2001, Experiment 3), or to encode the instrumental action-outcome contingencies (Corbit et al., 2001, Experiment 2). More recently, it was demonstrated that these effects are likely to be mediated by $\mu$-opioid processes. Infusion of the $\mu$-opioid receptor antagonist CTAP into the core immediately before the choice test disrupts outcome devaluation (Laurent et al., 2012). Importantly, it has also been demonstrated that inputs from the BLA to the NAc core are necessary for successful outcome devaluation. Rats with pretraining excitotoxic lesions of the BLA in one hemisphere and the NAc core in the contralateral hemisphere failed to show outcome devaluation, but showed normal acquisition of instrumental responding and intact specific Pavlovian-to-instrumental transfer (Shiflett and Balleine, 2010). The present results indicate that the functional interaction between the BLA and the NAc core may be mediated by the IC or, more conservatively, that the control of instrumental performance by the NAc core requires both BLA and IC inputs.

In addition to the core, the pDMS has also been heavily implicated in the performance of goal-directed actions. For example, outcome devaluation is also abolished in rats with pretraining or posttraining lesions of the pDMS and also in rats that receive a bilateral infusion of muscimol in the pDMS before test (Yin et al., 2005). Moreover, the performance of instrumental goal-directed actions is associated with increased extracellular signal-regulated kinase (ERK) in the pDMS and disruption of ERK activation prevents the performance of goal-directed actions guided by previously acquired associations (Shiflett et al., 2010). It seems plausible, therefore, that the IC could interact with the pDMS to guide performance based on the current incentive value of the outcome. Indeed, as disconnection studies do not provide evidence on the nature of connections (direct or otherwise), a potential IC-pDMS pathway cannot be ruled out. However, connections between the IC and the dorsal striatum appear to be significantly weaker than those between the IC and the ventral striatum (Wright and Groenenwegen, 1996; Reynolds and Zahm, 2005). Infusion of the retrograde neuronal tracers, Fluro-Gold or cholera toxin $\beta$ in the NAc core results in stronger labeling in the IC than an infusion in the caudate-putamen or NAc shell. This appears particularly true in the anterior IC (bregma +2.0 to +0.5$)$ however some retrograde labeling exists in the posterior IC (bregma -1.5 to -3.8 ) after injection more dorsally (Reynolds and Zahm, 2005). It seems likely, therefore, that communication between the IC and the NAc core, and not the pDMS, is the more critical for choice performance based on changes incentive value. Moreover, a 'feedforward' flow of information from more limbic regions of the striatum, such as the NAc shell and core, to the 'associative' DMS and then to the 'sensorimotor' dorsolateral striatum has been proposed (Haber et al., 2000), providing a potential circuit via which information from the IC, transferred to the core, influences goal-directed behavior produced by the pDMS.

To control performance, information about the incentive value of the outcome has to be integrated with information regarding the action-outcome contingency to control perfor- 
mance. Acquisition of the action-outcome contingency appears to require BLA input to the pDMS (Corbit et al., 2013), but also to the medial prefrontal cortex (mPFC). Specifically, rats with lesions of the prelimbic region (PL) of the mPFC fail to show outcome devaluation (Killcross and Coutureau, 2003; Ostlund and Balleine, 2005; Tran-Tu-Yen et al., 2009) and do not reduce their instrumental responding during contingency degradation (Balleine and Dickinson, 1998; Corbit and Balleine, 2003). Critically, lesions of the PL made after instrumental acquisition have no effect on outcome devaluation (Ostlund and Balleine, 2005; Tran-Tu-Yen et al., 2009; Gourley et al., 2010). At present, it is unknown whether the region of the IC that has been implicated in incentive memory is connected to the PL. It had previously been reported that the PL does not project to the IC (Allen et al., 1991), but a comprehensive study by Gabbott et al. (2003) provided evidence that there are reciprocal connections between the PL and the IC. However, the strongest identified projections from the PL to the IC are more anterior to the insular region investigated both here and in previous studies (Balleine and Dickinson, 2000; Parkes and Balleine, 2013). Therefore, it remains to be determined whether communication between the prefrontal and IC is necessary for goal-directed action.

We have previously implicated the BLA and the IC in the encoding and retrieval of incentive memory, respectively (Parkes and Balleine, 2013). Specifically, the BLA updates and encodes changes in incentive value during the devaluation phase in the IC for the retrieval of this value during the choice test. Here, we have shown that communication between the IC and the NAc core is critical during the choice test. Interestingly, a similar pathway has already been described in the drug addiction literature (Naqvi and Bechara, 2010). It has been proposed that the IC integrates information about the internal representation of drug effects received from the ventromedial prefrontal cortex with information about environmental cues received from the anterior cingulate cortex. In this view, this integrated information is finally sent from the IC to the NAc to guide the motivational effects of drugseeking behavior (Naqvi and Bechara, 2010; Kutlu et al., 2013). Here, we have proposed that goal-directed behavior is mediated by a similar, three-node serial pathway; that is, that encoding and retrieval are dependent on the BLA-IC pathway and retrieval and performance depend on the IC-core pathway.

\section{References}

Allen GV, Saper CB, Hurley KM, Cechetto DF (1991) Organization of visceral and limbic connections in the insular cortex of the rat. J Comp Neurol 311:1-16. Medline

Balleine BW, Dickinson A (1998) The role of incentive learning in instrumental revaluation by specific satiety. Animal Learning \& Behavior 26: 46-59. CrossRef

Balleine BW, Dickinson A (2000) The effect of lesions of the insular cortex on instrumental conditioning: evidence for a role in incentive memory. J Neurosci 20:8954-8964. Medline

Burton AC, Nakamura K, Roesch MR (2015) From ventral-medial to dorsal-lateral striatum: neural correlates of reward-guided decisionmaking. Neurobiol Learn Mem 117:51-59. Medline

Chikama M, McFarland NR, Amaral DG, Haber SN (1997) Insular cortical projections to functional regions of the striatum correlate with cortical cytoarchitecture organization in the primate. J Neurosci 17:9686-9705. Medline

Corbit LH, Balleine BW (2003) The role of prelimbic cortex in instrumental conditioning. Behav Brain Res 146:145-157. CrossRef Medline

Corbit LH, Muir JL, Balleine BW (2001) The role of the nucleus accumbens in instrumental conditioning: evidence of a functional dissociation between accumbens core and shell. J Neurosci 21:3251-3260. Medline

Corbit LH, Leung BK, Balleine BW (2013) The role of the amygdala-striatal pathway in the acquisition and performance of goal-directed instrumental actions. J Neurosci 33:17682-17690. CrossRef Medline

Dickinson A, Balleine BW (1994) Motivational control of goal-directed action. Animal Learning \& Behavior 22:1-18. CrossRef

Gabbott PL, Warner TA, Jays PR, Bacon SJ (2003) Areal and synaptic interconnectivity of prelimbic (area 32), infralimbic (area 25) and insular cortices in the rat. Brain Res 993:59-71. CrossRef Medline

Gourley SL, Lee AS, Howell JL, Pittenger C, Taylor JR (2010) Dissociable regulation of instrumental action within mouse prefrontal cortex. Eur J Neurosci 32:1726-1734. CrossRef Medline

Haber SN, Fudge JL, McFarland NR (2000) Striatonigrostriatal pathways in primates form an ascending spiral from the shell to the dorsolateral striatum. J Neurosci 20:2369-2382. Medline

Kelley AE, Domesick VB, Nauta WJ (1982) The amygdalostriatal projection in the rat-an anatomical study by anterograde and retrograde tracing methods. Neuroscience 7:615-630. CrossRef Medline

Killcross S, Coutureau E (2003) Coordination of actions and habits in the medial prefrontal cortex of rats. Cereb Cortex 13:400-408. CrossRef Medline

Kutlu MG, Burke D, Slade S, Hall BJ, Rose JE, Levin ED (2013) Role of insular cortex $D_{1}$ and $D_{2}$ dopamine receptors in nicotine selfadministration in rats. Behav Brain Res 256:273-278. CrossRef Medline

LaGraize SC, Fuchs PN (2007) GABAA but not GABAB receptors in the rostral anterior cingulate cortex selectively modulate pain-induced escape/avoidance behavior. Exp Neurol 204:182-194. CrossRef Medline

Laurent V, Leung B, Maidment N, Balleine BW (2012) $\mu$ - and $\delta$-opioidrelated processes in the accumbens core and shell differentially mediate the influence of reward-guided and stimulus-guided decisions on choice. J Neurosci 32:1875-1883. CrossRef Medline

McDonald AJ (1991) Topographical organization of amygdaloid projections to the caudatoputamen, nucleus accumbens, and related striatal-like areas of the rat brain. Neuroscience 44:15-33. CrossRef Medline

Naqvi NH, Bechara A (2009) The hidden island of addiction: the insula. Trends Neurosci 32:56-67. CrossRef Medline

Naqvi NH, Bechara A (2010) The insula and drug addiction: an interoceptive view of pleasure, urges, and decision-making. Brain Struct Funct 214:435-450. CrossRef Medline

Ostlund SB, Balleine BW (2005) Lesions of the medial prefrontal cortex disrupt the acquisition but not the expression of goal-directed learning. J Neurosci 25:7763-7770. CrossRef Medline

Parkes SL, Balleine BW (2013) Incentive memory: evidence the basolateral amygdala encodes and the insular cortex retrieves outcome values to guide choice between goal-directed actions. J Neurosci 33:8753-8763. CrossRef Medline

Paxinos G, Watson C (2007) The rat brain atlas in stereotaxic coordinates, Ed 6. San Diego: Academic.

Reynolds SM, Zahm DS (2005) Specificity in the projections of prefrontal and insular cortex to ventral striatopallidum and the extended amygdala. J Neurosci 25:11757-11767. CrossRef Medline

Shiflett MW, Balleine BW (2010) At the limbic-motor interface: disconnection of the basolateral amygdala from nucleus accumbens core and shell reveals dissociable components of incentive motivation. Eur J Neurosci 32:1735-1743. CrossRef Medline

Shiflett MW, Brown RA, Balleine BW (2010) Acquisition and performance of goal-directed instrumental actions depends on ERK signaling in distinct regions of dorsal striatum in rats. J Neurosci 30:2951-2959. CrossRef Medline

Soderman AR, Unterwald EM (2008) Cocaine reward and hyperactivity in the rat: sites of mu opioid receptor modulation. Neuroscience 154:15061516. CrossRef Medline

Tran-Tu-Yen DA, Marchand AR, Pape JR, Di Scala G, Coutureau E (2009) Transient role of the rat prelimbic cortex in goal-directed behaviour. Eur J Neurosci 30:464-471. CrossRef Medline

Trezza V, Damsteegt R, Achterberg EJ, Vanderschuren LJ (2011) Nucleus accumbens $\mu$-opioid receptors mediate social reward. J Neurosci 31: 6362-6370. CrossRef Medline

Wright CI, Groenewegen HJ (1996) Patterns of overlap and segregation between insular cortical, intermediodorsal thalamic and basal amygdaloid afferents in the nucleus accumbens of the rat. Neuroscience 73:359-373. CrossRef Medline

Yin HH, Ostlund SB, Knowlton BJ, Balleine BW (2005) The role of the dorsomedial striatum in instrumental conditioning. Eur J Neurosci 22: 513-523. CrossRef Medline 